\title{
Inter-relações de nitrogênio e fósforo na capacidade de combinação e na seleção em milho ${ }^{1}$
}

\author{
Leandro Vagno de Souza², Glauco Vieira Miranda ${ }^{3}$, João Carlos Cardoso Galvão ${ }^{3}$, Rodrigo Oliveira DeLima 4 , \\ Lauro José Moreira Guimarães ${ }^{5}$, Fernando Roberto Eckert ${ }^{6}$, Éder Eduardo Mantovani ${ }^{7}$
}

\section{RESUMO}

Tem sido observada, em milho, a redução da absorção de nitrogênio (N), por causa da deficiência de fósforo (P), mas o efeito dessa combinação na seleção de genitores ainda precisa de estudos. Os objetivos deste trabalho foram avaliar os efeitos das inter-relações de P e $\mathrm{N}$ na capacidade de combinação e seleção de genitores de milho. As 28 combinações híbridas $(\mathrm{CH})$, obtidas de um dialelo completo entre oito genótipos de milho e três testemunhas, foram avaliadas em quatro ambientes, obtidos pelas combinações de alta e baixa disponibilidade de P e N. Avaliaram-se o rendimento de grãos (RG) e as características secundárias: altura de plantas (AP) e espigas (AE), índice de colheita (IC), peso volumétrico (PV), prolificidade (PRL) e massa seca da parte aérea (MSPA). O efeito do estresse de N no RG foi similar em baixo e alto P. O genótipo P3041 apresentou alto RG em todos os ambientes. As correlações genéticas das características secundárias com o RG foram afetadas pelos ambientes. Em alto P, a capacidade geral de combinação (CGC) foi significativa para RG apenas em alto N. Em baixo P, a CGC apresentou significância em ambos os níveis de N. Os genótipos AG1051 e P3041 tiveram estimativas positivas de CGC em todos os ambientes. Conclui-se que, para o rendimento de grãos, a CGC somente não é importante em alto P e baixo N e a CEC não é importante em estresse de $\mathrm{P}$ ou $\mathrm{N}$.

Palavras-chave: Estresses abióticos, melhoramento, dialelo, Zea mays L.

\section{ABSTRACT}

\section{Nitrogen and phosphorus interrelationships for combining ability and selection in maize}

Phosphorus (P) deficiency has been reported to reduce nitrogen uptake $(\mathrm{N})$ in maize, but the effect of this combination for parent selection still needs further studies. The objective of this work was to evaluate the interrelationship between $\mathrm{P}$ and $\mathrm{N}$ in the combining ability and parent selection in maize. Twent-eight hybrid combinations $(\mathrm{CH})$, obtained from a complete diallel among eight maize genotypes, and three controls were evaluated in four environments characterized by a combination of high and low $\mathrm{P}$ and $\mathrm{N}$ availability. Grain yield (GY) and the secondary traits plant height (PH), ear height (EH), harvest index (HI), volumetric weight (VW), prolificacy (PRL) and dry weight of aerial parts (DWAP) were evaluated. The effect of nitrogen stress on GY was similar in the conditions of low and high P. The genotype P3041 showed high GY in all environments. Genetic correlations among secondary traits with GY were affected by all

\footnotetext{
Recebido para publicação em julho de 2008 e aprovado em agosto de 2010

${ }^{1}$ Parte da tese de Doutorado do primeiro autor apresentado ao Programa de Pós-Graduação em Genética e Melhoramento da UFV.

${ }^{2}$ Engenheiro-Agrônomo, Doutor. Dow Agrosciences Industria Ltda, Rodovia Anhanguera, s/n, km 344, Zona Rural, 14680-000, Ribeirão Preto, SP, Brasil. lvsouza2@dow.com ${ }^{3}$ Engenheiros-Agrônomos, Doutores. Universidade Federal de Viçosa, Departamento de Fitotecnia, Av. Peter Henry Rolfs, s/n, 36570-000, Viçosa, MG, Brasil. glaucovmiranda@ufv.br, jgalvao@ufv.br

${ }^{4}$ Engenheiro-Agrônomo, Mestre. Universidade Federal de Viçosa, Departamento de Fitotecnia, Av. Peter Henry Rolfs, s/n, 36570-000, Viçosa, MG, Brasil. rodrigoodelima@gmail.com ${ }^{5}$ Engenheiro Agrônomo, Doutor. Empresa Brasileira de Pesquisa Agropecuária, Centro Nacional de Pesquisa de Milho e Sorgo. Rodovia Estadual MG 424 km 45, $35701-970$, Sete Lagoas, MG, Brasil. lauro@cnpms.embrapa.br

${ }^{5}$ Engenheiro Agrônomo, Mestre. North Dakota State University, Fargo, Estados Unidos da América do Norte, fernandoagro2001@yahoo.com.br

7 Engenheiro Agrônomo. North Dakota State University, Fargo, Estados Unidos da América do Norte, dumantovani@yahoo.com.br
} 
environments. In high P, the general combining ability (GCA) for GY was significant only in high N. In low P, GCA of GY showed significance at both N levels. The genotypes AG1051 and P3041 had positives estimates of GCA for GY in all environments. It was conclude that for grain yield, the GCA alone is not important at high P and low $\mathrm{N}$ and the specific combing ability is not important at low $\mathrm{N}$ or $\mathrm{P}$.

Key-words: Abiotic stresses, breeding, diallel, Zea mays L.

\section{INTRODUÇÃO}

A seleção de genitores é uma das etapas primordiais em um programa de melhoramento de plantas e o cruzamento dialélico é um dos principais métodos para a identificação de genitores em melhoramento de milho (Machado et al., 2008; Miranda et al., 2008; Rodrigues et al., 2009;). Na análise de dialelos, é definido o efeito de capacidade geral de combinação (CGC), relacionado com os efeitos genéticos aditivos e com a frequência de genes favoráveis dos genitores e também com a capacidade específica de combinação (CEC), que estão associados à dominância, à epistasia e aos desvios do que seria esperado em relação à capacidade geral de combinação de dois genitores em combinações híbridas específicas (Sprague \& Tatum, 1942).

O desenvolvimento de cultivares de milho para maximizar o desempenho agronômico deve capitalizar a interação genótipos $\mathrm{x}$ ambientes, principalmente para as várias condições edafoclimáticas e sociais dos sistemas de produção (Miranda et al., 2009; Souza et al., 2009).

A baixa fertilidade natural dos solos de cerrado do Brasil é um limitante para o aumento na produção agrícola do país. A deficiência de fósforo (P) faz com este e o nitrogênio sejam os nutrientes mais usados na adubação, sendo que o nitrogênio $(\mathrm{N})$ é quantitativamente o nutriente mineral mais importante para o crescimento e desenvolvimento das plantas (Presterl et al., 2002).

Deficiência de P, reduzindo a absorção de N, tem sido observada na literatura (Novais \& Smyth, 1999). Plantas de milho com o sistema radicular dividido e alocado em vasos com duas disponibilidades de fósforo apresentaram sintomas de deficiência de nitrogênio em um dos lados da folha, em relação à nervura central o que mostra a inter- relação para esses dois nutrientes (Novais et al., 1985). Alves et al. (1996) observaram redução expressiva nos teores de $\mathrm{N}$ total nas folhas de milho, cultivadas em solução nutritiva, quando submetidas à omissão de $\mathrm{P}$, embora a solução nutritiva contivesse concentrações adequadas de nitrogênio para o desenvolvimento da planta. Assim, esses resultados evidenciaram a importância de considerar-se conjuntamente a eficiência nutricional para N e P nos critérios de avaliação e seleção de genótipos em um programa de melhoramento de milho.
Trabalhos realizados em milho tropical demonstraram ser possível o melhoramento para estresse de nitrogênio (Banziger \& Betran, 1997; Silva et al., 2008; Fidelis et al., 2007). A capacidade específica de combinação de linhagens endogâmicas de milho, em trabalho realizado por Betran et al. (2003a), avaliada em estresses de nitrogênio, foi negativa para híbridos, envolvendo linhagens endogâmicas originadas do mesmo germoplasma, e positiva e elevada para linhagens oriundas de fontes diferentes, demonstrando a importância da adoção de critérios que permitam a discriminação dos genitores, formando grupos divergentes para a extração de linhagens. Presterl et al. (2003) usaram dados de 21 experimentos com cultivares de milho, conduzidos em doses contrastantes de $\mathrm{N}$, para estimar parâmetros genéticos que otimizassem os programas de melhoramento a aumentar o rendimento de grãos sob baixo N. Os autores demonstraram que é possível selecionar genótipos de milho sob baixo N, embora haja uma redução no rendimento de grãos. E que, apesar dessa redução, a seleção direta, no ambiente de estresse, é mais eficiente que a seleção indireta em alto $\mathrm{N}$.

A herdabilidade para rendimento de grãos é menor em condições de baixo N (Presterl et al., 2003), levando a menores ganhos com melhoramento; assim, o uso de características secundárias em programa de melhoramento de milho para estas condições aumenta a eficiência de seleção de genótipos superiores (Betran et al., 2003b; Bänziger \& Lafite, 1997). Porém, o uso dessas características secundárias em programas de melhoramento de plantas é limitado pelo alto número de genótipos avaliados anualmente (Presterl et al., 2002). Por outro lado, Miranda et al. (2005a) descartaram linhagens de milho com menor eficiência no uso de nitrogênio, com base na leitura do clorofilômetro, por ocasião do florescimento das plantas. Assim, a importância das características secundárias é decorrência da correlação genética com as características de interesse econômico, na maioria das vezes rendimento de grãos.

Por estas razões, os objetivos deste trabalho foram identificar o controle genético de características agronômicas de milho, em ambientes contrastantes quanto à aplicação nitrogênio e fósforo e avaliar os efeitos das interrelações de N e P na seleção de genitores superiores. 


\section{MATERIAL E MÉTODOS}

No primeiro semestre de 2003, foi realizado o dialelo completo entre oito cultivares comerciais de milho, oriundos de diferentes instituições. Uma breve descrição desses cultivares é apresentada na Tabela 1.

As 28 combinações híbridas obtidas e as três testemunhas (DKB 747, DKB 333 e P 3041) foram avaliadas em quatro ambientes, em combinações de alto e baixo nitrogênio $(\mathrm{N})$ e fósforo $(\mathrm{P})$. A diferença entre os ambientes foi devida a combinações de doses (alta e baixa) de nitrogênio e fósforo. Os experimentos foram conduzidos no ano agrícola 2004/2005, na Estação Experimental de Coimbra, Coimbra - MG (latitude sul de 20 50’ 30" e longitude oeste de $42^{\circ} 48^{\prime} 30$ ", com altitude de 720 metros), pertencente ao Departamento de Fitotecnia da Universidade Federal de Viçosa. O delineamento experimental utilizado foi de blocos ao acaso, com duas repetições. Cada unidade experimental foi constituída por duas linhas de 5 metros de comprimento, espaçadas em 0,80 m, perfazendo uma área útil de $8 \mathrm{~m}^{2}$.

Em alto nitrogênio e fósforo, as doses utilizadas foram as recomendadas para a cultura do milho, com rendimento de grãos esperado superior a $8000 \mathrm{~kg} \mathrm{ha}^{-1}$, segundo a recomendação para o uso de corretivos e fertilizantes em Minas Gerais - $5^{\mathrm{a}}$ Aproximação (Alves et al., 1999). De acordo com os resultados da análise química do solo, este foi classificado como de muito baixa e média disponibilidade de fósforo e potássio, respectivamente. Dessa forma, as doses fornecidas em altos $\mathrm{N} \mathrm{e}$ $\mathrm{P}$, foram de $150 \mathrm{~kg} \mathrm{ha}^{-1}$ de nitrogênio e $120 \mathrm{~kg} \mathrm{ha}^{-1}$ de $\mathrm{P}_{2} \mathrm{O}_{5}$. Em baixos $\mathrm{N}$ e P, foram aplicados $30 \mathrm{~kg} \mathrm{ha}^{-1}$ de $\mathrm{Ne}$ $30 \mathrm{~kg} \mathrm{ha}^{-1}$ de $\mathrm{P}_{2} \mathrm{O}_{5}$. A adubação utilizada em baixos $\mathrm{N}$ e $\mathrm{P}$ foi inferior à recomendada para a menor faixa esperada de rendimento de grãos, segundo Ribeiro et al. (1999). A recomendação de potássio não variou entre os ambientes, sendo aplicados $80 \mathrm{~kg} \mathrm{ha}^{-1}$ de $\mathrm{K}_{2} \mathrm{O}$ em todos os ambientes. Os demais tratos culturais foram realizados sempre que necessários, de acordo com as recomendações técnicas para a cultura do milho (Galvão \& Miranda, 2004).
As seguintes características foram avaliadas: rendimento de grãos (RG), expresso em $\mathrm{kg} \mathrm{ha}^{-1}$; altura de plantas (AP) e de espigas (AE), medida em centímetros, em cinco plantas competitivas por parcela, após o florescimento masculino; prolificidade (PRL), obtida pelo número médio de espigas por planta; peso volumétrico (PV), expresso em $\mathrm{kg} \mathrm{m}^{-3}$ de grãos; peso de 100 grãos, expresso em gramas; produção de matéria seca da parte aérea (MSPA), expressa em t ha ${ }^{-1}$ e o índice de colheita (IC), estimado pela razão entre rendimento de grãos e MSPA e expresso em percentagem. A MSPA foi estimada após a maturidade fisiológica dos grãos, da seguinte maneira: cinco plantas competitivas foram coletadas por parcela, separando-se o grão da parte aérea (caule + folhas + palha + sabugo) e em seguida efetuando-se a pesagem da parte aérea. Após a pesagem retirou-se uma amostra de 1000 gramas, que foi submetida à secagem em estufa a $70^{\circ} \mathrm{C}$, até atingir peso constante.

O teste $\mathrm{t}$ foi utilizado para comparações múltiplas, com nível de significância de $5 \%$ de probabilidade. Sendo a DMS dada por:

$D M S=t_{(v, \alpha / 2)} \sqrt{\hat{V}(D)}=t_{(v, \alpha / 2)} \sqrt{\frac{2 Q M E}{r}}$

O método utilizado para estimar os efeitos de capacidade geral e específica de combinação foi a proposta por Griffing (1956), método 4, utilizando-se apenas as combinações híbridas. As análises dialélicas foram realizadas em cada ambiente, utilizando o seguinte modelo:

$Y_{i j}=\mu+g_{i}+g_{j}+s_{i j}+\bar{\varepsilon}_{i j}$

em que:

$Y_{i j}$ : valor médio do híbrido ij (i, j =1, 2, 3... p, i < j); $\mu$ :média geral;

$g_{i}, g_{j}$ : efeitos da capacidade geral de combinação (CGC) do i-ésimo e j-ésimo genitor, respectivamente;

$S_{i j}$ : efeito da capacidade específica de combinação (CEC) para as combinações híbridas entre os genitores i e j; e

$\bar{\varepsilon}_{i j}$ : erro experimental médio.

Tabela 1. Características agronômicas dos genitores de milho utilizados no dialelo completo para obtenção das combinações híbridas

\begin{tabular}{lcccccc}
\hline Cultivar & Obtentor & Nível Tecnológico & Tipo $^{\mathbf{1}}$ & Ciclo & Co $^{2}$ & Tipo de grão \\
\hline AG 1051 & Monsanto/Agroceres & Médio/alto & HD & Semiprecoce & AM & Dentado \\
AG 122 & Monsanto/Agroceres & Médio & HD & Precoce & AM/AL & Semidentado \\
BR 106 & Embrapa & Baixo/médio & V & Semiprecoce & AM & Semidentado \\
DKB 747 & Monsanto/Dekalb & Alto & HD & Precoce & AL & Duro \\
DKB- 901 & Monsanto/Dekalb & Alto & HS & Superprecoce & AM & Dentado \\
DKB-333B & Monsanto/Dekalb & Alto & HS & Semiprecoce & AM/ÃL & Semi duro \\
P 3041 & Pioneer & Médio/alto & HT & Precoce & AL & Duro \\
XB 7012 & Semeali & Médio/alto & HT & Precoce & LR & Duro \\
\hline
\end{tabular}

${ }^{1} \mathrm{HD}=$ híbrido duplo, HS=híbrido simples, $\mathrm{HT}=$ =híbrido triplo e $\mathrm{V}=$ variedade; ${ }^{2} \mathrm{AM}=$ amarelo, $\mathrm{AM} / \mathrm{AL}=$ =amarelo alaranjado, LR=laranja. 
Todas as análises estatísticas foram realizadas com auxílio do Aplicativo Computacional em Genética e Estatística - Programa Genes versão Windows (Cruz, 2006).

\section{RESULTADOS E DISCUSSÃO}

Em baixo fósforo $(\mathrm{P})$ e com alto nitrogênio $(\mathrm{N})$ a média de rendimento de grãos (RG) foi de $8470 \mathrm{~kg} \mathrm{ha}^{-1}$, variando de 5196 a $11976 \mathrm{~kg} \mathrm{ha}^{-1}$ (Tabela 2). Com baixos níveis de P e N a média de RG foi igual a $6249 \mathrm{~kg} \mathrm{ha}^{-1}$, variando de 4276 a 10184 kg ha-1 entre os genótipos avaliados. Em altos $\mathrm{P}$ e $\mathrm{N}$, ambientes sem estresse nutricional, a média de RG foi de $10121 \mathrm{~kg} \mathrm{ha}^{-1}$, e em alto $\mathrm{P}$ e baixo $\mathrm{N}$ igual a 7 $766 \mathrm{~kg} \mathrm{ha}^{-1}$ (Tabela 2). As médias obtidas nos quatro ambientes evidenciaram o potencial produtivo dos genótipos, que mesmo em baixos N e P, alcançaram média superior a
$6000 \mathrm{~kg} \mathrm{ha}^{-1}$, utilizando-se adubação recomendada para RG esperada de até $4000 \mathrm{~kg} \mathrm{ha}^{-1}$. Esses resultados mostraram que em baixo $\mathrm{N}$ os RG foram semelhantes tanto em baixo P (-26,22 \%) quanto em alto P (-23,27\%).

O genótipo comercial, o híbrido triplo $\mathrm{P}$ 3041, usado com testemunha, apresentou maior RG que os demais genótipos, em quase todos os ambientes, com exceção de alto P e baixo N. Neste ambiente, este genótipo foi superado apenas pela combinação híbrida $(\mathrm{CH})$ XB 7012 x DKB 333 , sem dela diferir estatisticamente, pela DMS- $t_{5 \%}$, . Esses resultados evidenciam a ampla adaptação do hibrido triplo P 3041, concordando com os resultados encontrados por Miranda et al. (2005b). Os autores ao avaliarem 15 cultivares de milho de ciclo precoce em dois anos e três locais, no Estado de Minas Gerais verificaram que ape-

Tabela 2. Média de rendimento de grãos $\left(\mathrm{kg} \mathrm{ha}^{-1}\right)$ dos genótipos de milho avaliados em quatro ambientes obtidos pelas combinações de alto e baixo P e N

\begin{tabular}{|c|c|c|c|c|}
\hline \multirow{2}{*}{ Genótipos } & \multicolumn{2}{|c|}{ Baixo P } & \multicolumn{2}{|c|}{ Alto $\mathbf{P}$} \\
\hline & Alto $\mathbf{N}$ & Baixo N & Alto $\mathbf{N}$ & Baixo $\mathbf{N}$ \\
\hline AG 1051 x DKB 901 & 11749 & 9435 & 13316 & 8544 \\
\hline AG 1051 x XB 7012 & 9023 & 6008 & 9999 & 8355 \\
\hline AG 1051 x DKB 333 & 8389 & 6761 & 8894 & 6549 \\
\hline AG 1051 x P 3041 & 10630 & 9696 & 8335 & 8217 \\
\hline AG 1051 x BR 106 & 8690 & 6120 & 9937 & 7626 \\
\hline AG 1051 x DKB 747 & 8049 & 7556 & 10852 & 8034 \\
\hline AG 1051 x AG 122 & 9520 & 5888 & 11908 & 8046 \\
\hline DKB 901 x XB 7012 & 8584 & 5838 & 5912 & 5727 \\
\hline DKB 901 x DKB 333 & 9047 & 6253 & 10027 & 8373 \\
\hline DKB 901 x P 3041 & 7805 & 6288 & 9649 & 7096 \\
\hline DKB 901 x BR 106 & 8570 & 7010 & 8437 & 5786 \\
\hline DKB 901 x DKB 747 & 7301 & 5853 & 8675 & 7462 \\
\hline DKB 901 x AG 122 & 10326 & 6714 & 5934 & 7618 \\
\hline XB 7012 x DKB 333 & 7014 & 4592 & 9458 & 10912 \\
\hline XB 7012 x P 3041 & 7800 & 5570 & 10984 & 8359 \\
\hline XB 7012 x BR 106 & 5939 & 4737 & 10505 & 7278 \\
\hline XB 7012 x DKB 747 & 6958 & 4780 & 10326 & 6029 \\
\hline XB 7012 x AG 122 & 9601 & 6762 & 10723 & 6797 \\
\hline DKB 333 x P 3041 & 10143 & 6980 & 12184 & 10435 \\
\hline DKB 333 x BR 106 & 8299 & 4419 & 9178 & 5668 \\
\hline DKB 333 x DKB 747 & 6487 & 5414 & 10140 & 7581 \\
\hline DKB 333 x AG 122 & 6610 & 4276 & 10112 & 8426 \\
\hline P 3041 x BR 106 & 9260 & 5220 & 10193 & 9667 \\
\hline P 3041 x DKB 747 & 7993 & 5697 & 10584 & 6986 \\
\hline P 3041 x AG 122 & 8489 & 6296 & 11537 & 8217 \\
\hline BR 106 x DKB 747 & 5196 & 5551 & 10579 & 7789 \\
\hline BR 106 x AG 122 & 7237 & 5355 & 8380 & 6606 \\
\hline DKB 747 x AG 122 & 7731 & 6042 & 11527 & 7543 \\
\hline DKB 747 & 8538 & 6910 & 9987 & 7321 \\
\hline DKB 333 & 9618 & 5507 & 10034 & 6797 \\
\hline P 3041 & 11976 & 10184 & 15466 & 10903 \\
\hline Média & 8470 & 6249 & 10121 & 7766 \\
\hline DMS- $t_{5 \%}$ & 3066 & 2932 & 2495 & 3296 \\
\hline CV\% & 17,7 & 22,9 & 12,0 & 20,7 \\
\hline
\end{tabular}

Rev. Ceres, Viçosa, v. 57, n.5, p. 633-641, set/out, 2010 
nas o cultivar P 3041 superou as médias de rendimento de grãos em todos os experimentos.

Apesar do híbrido triplo P 3041, superar todos os genótipos, em RG, em três ambientes, nestes ambientes, houve $\mathrm{CHs}$ que não diferiram estatisticamente desta testemunha pela DMS- t $_{5 \%}$. E ainda, em todos os ambientes houve $\mathrm{CH}$ que apresentaram desempenho superior às duas outras testemunhas, o híbrido simples DK 333 e o híbrido duplo DKB 747. Esses resultados mostram o potencial produtivo destas $\mathrm{CH}$, nos diferentes ambientes.

A precisão experimental, medida pelo coeficiente de variação experimental, foi superior nos ambientes de estresse; no entanto, os valores encontrados para todas as características estão dentro dos valores considerados baixos ou médios em experimentação com a cultura de milho (Scapim, 1995).

As médias das características secundárias AP, AE, PRL e PV quase não foram afetadas pelos ambientes (Tabela 3), concordando com os resultados de Zhu et al. (2005). Eles verificaram que o estresse de P afetou com maior intensidade a característica principal, mas que as diferenças entre as médias das características secundárias entre os ambientes com e sem estresse de $\mathrm{P}$ não foram significativas.

As correlações genéticas das características secundárias com o RG foram afetadas pelos ambientes (Tabela 3). Em baixo P, as características apresentaram correlações genéticas com RG em mesmo sentido, em ambos os níveis de $\mathrm{N}$, embora haja diferenças nas magnitudes destas. Em alto P, nota-se maior associação das características secundárias com RG em baixo N, mostrada pelas maiores magnitudes das correlações genéticas, o que não é observado em condição de alto N. Esses resultados foram diferentes dos encontrados por Ribaut et al. (2007). Esses autores avaliaram uma população segregante de milho, em alto e baixo $\mathbf{N}$, e verificaram que as correlações fenotípica e genotípica de características secundárias com o RG foram pouco afetadas pelo estresse de N. No entanto, os mesmo autores observaram que, ao considerar outro estresse, neste caso seca, as estimativas das correlações foram afetadas tanto em magnitude quanto em sentido.

Por estas razões, para a utilização das características secundárias, como auxílio no processo seletivo em ambientes com e sem estresse de $\mathrm{N}$, torna-se necessário determinar a disponibilidade de $\mathrm{P}$, uma vez que essa disponibilidade altera a natureza e magnitude da correlação genética das características secundárias com o RG. Em condições ótimas de $\mathrm{P}$ e $\mathrm{N}$, as características secundárias são de pouca utilidade no processo seletivo em programas de melhoramento para este conjunto de genótipos, por causa das baixas magnitudes das suas correlações genéticas com RG.

Os resultados obtidos com a realização da análise dialélica mostraram que as inter- relações entre fósforo e nitrogênio afetaram a detecção de efeitos significativos de capacidade geral de combinação (CGC) e capacidade específica de combinação (CEC), ou seja, os ambientes afetaram o controle genético para a maioria das características estu-

Tabela 3. Média (m), valor da estatística F (F), coeficiente de variação experimental (CV\%) e correlações genéticas $\left(r_{\mathrm{g}}\right)$ entre rendimento de grãos e as características altura de planta $(\mathrm{AP}, \mathrm{cm})$, altura de espiga $(\mathrm{AE}, \mathrm{cm})$, prolificidade (PRL, número médio de espigas/planta), peso volumétrico (PV, $\mathrm{kg} \mathrm{m}^{-3}$ ), peso de 100 grãos (P100, gramas) e índice de colheita (IC, \%) obtidos em alto N (AN) e baixo $\mathrm{N}(\mathrm{BN})$ em condições de alto e baixo fósforo

\begin{tabular}{|c|c|c|c|c|c|c|c|c|}
\hline \multirow{3}{*}{ Característica } & \multicolumn{8}{|c|}{ Baixo P } \\
\hline & \multicolumn{2}{|c|}{$\mathbf{F}$} & \multicolumn{2}{|c|}{ CV (\%) } & \multicolumn{2}{|c|}{ m } & \multicolumn{2}{|c|}{$r_{g}$} \\
\hline & AN & BN & AN & BN & AN & BN & AN & BN \\
\hline$\overline{\mathrm{AP}}$ & $3,10^{* *}$ & $2,96^{* *}$ & 3,8 & 5,0 & 243 & 229 & $-0,55$ & $-0,91$ \\
\hline $\mathrm{AE}$ & $2,43^{* *}$ & $2,67^{* *}$ & 7,2 & 8,9 & 133 & 125 & $-0,49$ & $-0,67$ \\
\hline PRL & $1,68^{\text {ns }}$ & $0,61^{\mathrm{ns}}$ & 14,9 & 20,5 & 0,84 & 0,89 & 0,86 & 0,00 \\
\hline PV & $1,74^{\mathrm{ns}}$ & $2,04^{*}$ & 4,8 & 3,8 & 696 & 687 & $-0,97$ & $-0,85$ \\
\hline P100 & $1,73^{\text {ns }}$ & $1,74^{\mathrm{ns}}$ & 6,8 & 5,9 & 33 & 31 & 1,00 & 0,69 \\
\hline \multirow[t]{4}{*}{ IC } & $3,80^{* *}$ & $2,51^{* *}$ & 14,1 & 21,0 & 41 & 34 & 0,83 & 0,90 \\
\hline & \multicolumn{8}{|c|}{ Alto P } \\
\hline & \multicolumn{2}{|c|}{$\mathbf{F}$} & \multicolumn{2}{|c|}{ CV (\%) } & \multicolumn{2}{|c|}{$\mathbf{M}$} & \multicolumn{2}{|c|}{$r_{g}$} \\
\hline & NA & BN & AN & BN & NA & BN & AN & BN \\
\hline$\overline{\mathrm{AP}}$ & $4,11^{* *}$ & $2,41^{* *}$ & 2,6 & 3,7 & 256 & 250 & 0,40 & 0,81 \\
\hline $\mathrm{AE}$ & $4,91^{* *}$ & $3,05^{* *}$ & 5,4 & 6,3 & 146 & 139 & 0,48 & 0,56 \\
\hline PRL & $0,78^{\text {ns }}$ & $0,95^{\mathrm{ns}}$ & 20,7 & 15,3 & 0,85 & 0,89 & 0,00 & 0,00 \\
\hline PV & $1,62^{\mathrm{ns}}$ & $1,13^{\mathrm{ns}}$ & 3,5 & 6,2 & 739 & 708 & $-0,07$ & 1,00 \\
\hline P100 & $0,94^{\mathrm{ns}}$ & $2,23^{*}$ & 6,5 & 5,7 & 32 & 30 & 0,00 & $-0,39$ \\
\hline IC & $1,71^{\mathrm{ns}}$ & $3,09^{* *}$ & 14,6 & 13,6 & 43 & 39 & $-0,13$ & $-0,27$ \\
\hline
\end{tabular}

**** significativo a 5 e $1 \%$ de probabilidade, respectivamente; ${ }^{\text {ns }}$ não significativo 
dadas nos genótipos de milho (Tabela 4). Resultados similares foram obtidos por Gama et al. (2006), que, ao estudarem a capacidade de combinação de linhagens de milho em ambientes com e sem estresses de fósforo, verificaram, para a maioria das características, que houve interação significativa da CGC e CEC com o ambiente.

O quadrado médio de CGC e CEC para característica RG não foi significativo no ambiente formado pela combinação de alto $\mathrm{P}$ e baixo $\mathrm{N}$, ou seja, neste ambiente não foram verificadas diferenças significativas entre os genótipos, tanto para os efeitos gênicos aditivos, como para os não aditivos. Assim, nenhum ganho será esperado para RG neste ambiente, caso alguma estratégia de melhoramento seja aplicada. Para este ambiente, os resultados foram distintos dos obtidos por Médice et al. (2004). Os autores avaliaram 15 híbridos simples de milho, obtidos de cinco linhagens endogâmicas em condições de alto e baixo $\mathrm{N}$, e concluíram que os efeitos genéticos aditivos são responsáveis pelo controle do rendimento de grãos, independentemente do nível N.

Tabela 4. Quadrados médios dos efeitos de capacidade geral de combinação (CGC) e capacidade específica de combinação (CEC) para as características rendimento de grãos (RG), altura de planta (AP), altura de espiga (AE), prolificidade (PRL), peso volumétrico (PV), peso de 100 grãos (P100) e índice de colheita (IC) das combinações de milho híbrido avaliadas nos quatro ambientes, formados pelas combinações de alto e baixo P e N

\begin{tabular}{|c|c|c|c|c|c|c|c|c|}
\hline \multirow{3}{*}{ Característica } & \multicolumn{4}{|c|}{ Baixo P } & \multicolumn{4}{|c|}{ Alto $\mathbf{P}$} \\
\hline & \multicolumn{2}{|c|}{ Alto $\mathbf{N}$} & \multicolumn{2}{|c|}{ Baixo N } & \multicolumn{2}{|c|}{ Alto $\mathbf{N}$} & \multicolumn{2}{|c|}{ Baixo N } \\
\hline & CGC & CEC & CGC & CEC & CGC & CEC & CGC & CEC \\
\hline RG & $10391998 * *$ & $2189602^{\mathrm{ns}}$ & $11934021^{* * *}$ & $2404902^{\mathrm{ns}}$ & $5011415^{* *}$ & $5494400 * *$ & $3524572^{\mathrm{ns}}$ & $3163639^{\mathrm{ns}}$ \\
\hline AP & $913,44 * *$ & $53,81^{\text {ns }}$ & $1987,49 * *$ & $144,70^{\text {ns }}$ & $514,76^{* *}$ & $81,12^{\text {ns }}$ & $570,90 * *$ & $108,58^{\mathrm{ns}}$ \\
\hline $\mathrm{AE}$ & $592,80 * *$ & $53,05^{\mathrm{ns}}$ & $1438,49 * *$ & $198,10^{\mathrm{ns}}$ & $726,33 * *$ & $109,00^{\mathrm{ns}}$ & $676,76^{* *}$ & $79,57^{\mathrm{ns}}$ \\
\hline PRL & $0,0424 *$ & $0,2024^{\mathrm{ns}}$ & $0,0480^{\mathrm{ns}}$ & $0,025^{\text {ns }}$ & $0,0171^{\mathrm{ns}}$ & $0,0218^{\mathrm{ns}}$ & $0,0191^{\mathrm{ns}}$ & $0,0174^{\mathrm{ns}}$ \\
\hline PV & $3621,23^{*}$ & $1645,54^{\mathrm{ns}}$ & $5324,38 * *$ & $924,81^{\text {ns }}$ & $3,7630^{\mathrm{ns}}$ & $0,7176^{\mathrm{ns}}$ & $3079,66^{\mathrm{ns}}$ & $2054,18^{\text {ns }}$ \\
\hline P100 & $16,23 * *$ & $5,24^{\mathrm{ns}}$ & $12,63 * *$ & $8,57^{*}$ & $5,8273^{\text {ns }}$ & $3,76^{\text {ns }}$ & $5,00^{\text {ns }}$ & $7,03 *$ \\
\hline IC & $375,97 * *$ & $62,28^{\mathrm{ns}}$ & $349,32 * *$ & $117,85^{*}$ & $122,66^{*}$ & $42,00^{\mathrm{ns}}$ & $109,75^{* *}$ & $89,75^{\mathrm{ns}}$ \\
\hline
\end{tabular}

*,** significativo a 5 e $1 \%$ de probabilidade, respectivamente; ${ }^{\text {ns }}$ não significativo

Tabela 5. Estimativas de efeitos médios de capacidade geral de combinação para as características rendimento de grãos (RG), altura de planta (AP), altura de espiga (AE), prolificidade (PRL), peso volumétrico (PV), peso de 100 grãos (P100) e índice de colheita (IC) dos oito genitores de milho do dialelo completo avaliados em quatro ambientes formados pelas combinações de alto e baixo $\mathrm{P}$ e $\mathrm{N}$

\begin{tabular}{|c|c|c|c|c|c|c|c|c|c|c|}
\hline \multicolumn{3}{|c|}{ Ambiente/característica } & \multirow{2}{*}{$\begin{array}{c}\text { AG 1051 } \\
1323,40\end{array}$} & \multirow{2}{*}{$\begin{array}{c}\text { DKB } 901 \\
878,56\end{array}$} & \multirow{2}{*}{$\begin{array}{c}\text { XB } 7012 \\
-531,85\end{array}$} & \multirow{2}{*}{$\begin{array}{c}\text { DKB } 333 \\
-353,52\end{array}$} & \multirow{2}{*}{$\begin{array}{l}\text { P 3041 } \\
668,31\end{array}$} & \multirow{2}{*}{$\begin{array}{r}\text { BR } 106 \\
-819,77\end{array}$} & \multirow{2}{*}{$\begin{array}{c}\text { DKB } 747 \\
-1399,10\end{array}$} & \multirow{2}{*}{$\begin{array}{c}\text { AG } 122 \\
233,98\end{array}$} \\
\hline \multirow{13}{*}{ 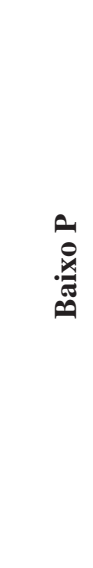 } & \multirow{7}{*}{$\begin{array}{l}\text { Z } \\
\stackrel{0}{u}\end{array}$} & RG & & & & & & & & \\
\hline & & AP & $-2,40$ & $-17,06$ & $-5,23$ & 4,10 & 3,02 & 8,77 & $-1,40$ & 10,19 \\
\hline & & $\mathrm{AE}$ & 0,83 & $-11,42$ & $-3,17$ & 0,75 & 3,50 & 7,42 & $-7,17$ & 9,25 \\
\hline & & PRL & 0,01 & $-0,02$ & $-0,01$ & $-0,01$ & 0,14 & $-0,05$ & $-0,08$ & 0,02 \\
\hline & & PV & $-27,94$ & $-9,85$ & 18,40 & 11,48 & $-0,69$ & 15,40 & 12,48 & $-19,27$ \\
\hline & & $\mathrm{P} 100$ & 1,21 & 1,88 & $-0,79$ & $-1,54$ & $-0,63$ & 0,21 & $-0,88$ & 0,54 \\
\hline & & IC & 2,64 & 9,81 & $-4,15$ & $-1,39$ & 0,88 & $-3,34$ & $-8,45$ & 4,00 \\
\hline & \multirow{6}{*}{ 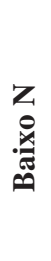 } & RG & 1447,69 & 768,94 & $-748,40$ & $-680,48$ & 494,85 & $-727,40$ & $-314,23$ & $-240,98$ \\
\hline & & AP & 0,06 & $-19,19$ & $-8,10$ & 2,40 & 1,48 & 13,06 & $-2,19$ & 12,48 \\
\hline & & $\mathrm{AE}$ & 4,77 & $-15,40$ & $-6,90$ & $-0,15$ & 3,35 & 12,19 & $-5,65$ & 7,77 \\
\hline & & PV & $-26,35$ & $-11,44$ & 6,90 & 9,40 & 20,56 & 3,98 & 16,73 & $-19,77$ \\
\hline & & P100 & 1,23 & 1,35 & $-0,50$ & $-0,57$ & $-0,71$ & 0,05 & $-0,59$ & $-0,26$ \\
\hline & & IC & 4,89 & 7,96 & $-2,08$ & $-5,26$ & $-0,19$ & $-4,03$ & $-1,09$ & $-0,20$ \\
\hline \multirow{8}{*}{$\begin{array}{l}\text { Z } \\
\stackrel{9}{2}\end{array}$} & \multirow{5}{*}{$\stackrel{0}{\stackrel{9}{2}}$} & RG & 611,69 & $-1270,15$ & $-277,48$ & 70,27 & 649,19 & $-393,81$ & 518,69 & 91,60 \\
\hline & & AP & 4,08 & $-11,83$ & $-5,50$ & $-0,08$ & 0,00 & 8,50 & $-1,42$ & 6,25 \\
\hline & & $\mathrm{AE}$ & 9,79 & $-10,13$ & $-4,71$ & $-5,21$ & $-0,54$ & 11,21 & $-5,21$ & 4,79 \\
\hline & & PV & $-27,19$ & $-1,19$ & 7,31 & 1,56 & 7,90 & 20,40 & 2,56 & $-11,35$ \\
\hline & & IC & 3,55 & 2,85 & 0,73 & $-6,76$ & 0,38 & $-0,66$ & 0,89 & $-0,99$ \\
\hline & \multirow{3}{*}{ 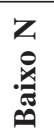 } & AP & 5,83 & $-9,33$ & $-8,00$ & 2,25 & 1,75 & 11,08 & $-4,08$ & 0,50 \\
\hline & & $\mathrm{AE}$ & 8,67 & $-9,92$ & $-7,00$ & $-2,42$ & 1,17 & 12,00 & $-4,25$ & 1,75 \\
\hline & & IC & $-0,59$ & 5,49 & $-0,76$ & $-3,74$ & 1,97 & $-3,76$ & 0,31 & 1,07 \\
\hline
\end{tabular}

Rev. Ceres, Viçosa, v. 57, n.5, p. 633-641, set/out, 2010 
Em baixo P, o quadrado médio de CGC foi significativo para quase todas as características estudadas nos dois níveis de $\mathrm{N}$, com exceção PRL em baixo $\mathrm{N}$, o que denota a existência de variabilidade genética, resultante dos efeitos gênicos aditivos no controle da expressão gênica para estas características. A disponibilidade de $\mathrm{P}$ afetou a variabilidade das características secundárias para efeitos aditivos, uma vez que, em alto P, estas apresentaram magnitudes de quadrado médio para CGC inferiores ao observado em baixo $\mathrm{P}$, nos dois níveis de N. Coimbra et al. (2008), avaliando a capacidade de combinação de cultivares de milho, em solos com baixo nível de fertilidade, encontraram significâncias para CGC e CEC para RG, mas não encontraram significância para PRL.

Em baixo P, os genitores que apresentaram estimativas positivas de CGC, para RG nos dois níveis de N, foram AG 1051, DKB 901 e P 3041 e o híbrido duplo AG 122, que apresentou CGC positiva somente em alto N (Tabela 5). Em alto $\mathrm{P}$ e alto $\mathrm{N}$, os genitores que apresentaram as maiores estimativas positivas de CGC foram AG 1051, P 3041, DKB 747, AG 122 e DKB 333. Com base na CGC em todos os ambientes, os genitores AG 1051 e P 3041 seriam os selecionados, pois apresentam estimativas positivas de CGC.

Quanto a CEC, a combinação híbrida AG 1051 x DKB 901 obteve a maior estimativa positiva de CEC e a maior média de RG (Tabela 6). Contudo, esta $\mathrm{CH}$ não diferiu das combinações híbridas AG 1051 x DKB 901, AG 1051 x AG 122, XB 7012 x P 3041, DKB 333 x P 3041, P 3041 x AG 122 e DKB 747 x AG 122. Estas combinações híbridas foram selecionadas por reunirem estimativas de CEC positivas para pelo menos um genitor com alta CGC e alto RG.

Para a seleção das melhores CH e, por consequência, para a identificação dos genitores com maior potencial para a extração de linhagens nos demais ambientes, onde não foram verificados efeitos significativos de CEC para RG, uma alternativa viável para auxiliar no processo de seleção seria a utilização das características secundárias,

Tabela 6. Estimativas de efeitos médios de capacidade específica de combinação para as características: índice de colheita (IC), peso de 100 grãos (P100) e rendimento de grãos (RG) de 28 combinações híbridas, obtidas de oito genitores em esquema de dialelo completo, avaliados nos ambientes de baixo $\mathrm{P}$ e baixo $\mathrm{N}$ e alto $\mathrm{P}$ e baixo $\mathrm{N}$ e alto $\mathrm{N}$

\begin{tabular}{|c|c|c|c|c|}
\hline \multirow{3}{*}{ Combinação Híbrida } & \multirow{2}{*}{\multicolumn{2}{|c|}{$\begin{array}{l}\text { Baixo } \mathbf{P} \\
\text { Baixo N }\end{array}$}} & \multicolumn{2}{|c|}{ Alto $\mathbf{P}$} \\
\hline & & & \multirow{2}{*}{$\begin{array}{c}\text { Baixo N } \\
\text { P100 }\end{array}$} & \multirow{2}{*}{$\begin{array}{c}\text { Alto } \mathbf{N} \\
\text { RG }\end{array}$} \\
\hline & IC & P100 & & \\
\hline AG 1051 x DKB 901 & $-2,08$ & $-0,57$ & $-1,85$ & 4035,98 \\
\hline AG 1051 x XB 7012 & $-3,50$ & 0,35 & $-0,68$ & $-273,69$ \\
\hline AG 1051 x DKB 333 & 0,17 & $-0,15$ & $-0,01$ & $-1726,94$ \\
\hline AG 1051 x P 3041 & 11,25 & 0,51 & 1,07 & $-2864,36$ \\
\hline AG 1051 x BR 106 & $-1,08$ & $-0,15$ & 0,24 & $-219,86$ \\
\hline AG 1051 x DKB 747 & 1,50 & 1,93 & 3,90 & $-216,86$ \\
\hline AG 1051 x AG 122 & $-6,25$ & $-1,90$ & $-2,68$ & 1265,73 \\
\hline DKB 901 x XB 7012 & 0,75 & $-0,32$ & 1,24 & $-2479,36$ \\
\hline DKB 901 x DKB 333 & 0,92 & $-2,32$ & $-1,10$ & 1288,39 \\
\hline DKB 901 x P 3041 & $-15,50$ & 0,35 & $-2,01$ & 331,48 \\
\hline DKB 901 x BR 106 & 3,17 & 0,18 & 1,65 & 161,98 \\
\hline DKB 901 x DKB 747 & 0,75 & $-1,74$ & $-1,68$ & $-512,02$ \\
\hline DKB 901 x AG 122 & 12,00 & 4,43 & 3,74 & $-2826,44$ \\
\hline XВ 7012 x DKB 333 & 0,00 & 0,60 & 1,57 & $-273,77$ \\
\hline XB 7012 x P 3041 & $-0,92$ & 0,26 & $-0,85$ & 673,31 \\
\hline XB 7012 x BR 106 & $-0,25$ & $-0,40$ & 2,18 & 1237,31 \\
\hline ХВ 7012 x DKB 747 & 0,33 & $-0,32$ & 0,49 & 145,81 \\
\hline XB 7012 x AG 122 & 3,58 & $-0,15$ & 0,40 & 970,39 \\
\hline DKB 333 x P 3041 & 9,25 & 1,26 & 1,32 & 1525,56 \\
\hline DKB 333 x BR 106 & $-2,08$ & 1,60 & 0,51 & $-436,94$ \\
\hline DKB 333 x DKB 747 & 0,00 & 0,68 & 0,35 & $-387,44$ \\
\hline DKB 333 x AG 122 & $-8,25$ & $-1,65$ & 0,93 & 11,14 \\
\hline P 3041 x BR 106 & 0,50 & 0,76 & 0,07 & $-0,86$ \\
\hline P 3041 x DKB 747 & $-4,92$ & $-2,15$ & 0,26 & $-522,86$ \\
\hline P 3041 x AG 122 & 0,33 & $-0,99$ & 0,65 & 857,73 \\
\hline BR 106 x DKB 747 & 1,75 & $-0,32$ & 0,10 & 515,14 \\
\hline BR 106 x AG 122 & $-2,00$ & $-1,65$ & 0,82 & $-1256,77$ \\
\hline DKB 747 x AG 122 & 0,58 & 1,93 & 2,01 & 978,23 \\
\hline
\end{tabular}


que apresentaram CEC significativa e que tenham correlação genética com o RG. Em alto P e baixo N, a característica P100 apresentou CEC significativa e as $\mathrm{CH}$ que apresentaram estimativas positivas de CEC e tiveram alto RG foram AG 1051 x P 3041, AG 1051 x BR 106, AG 1051 x DKB 747, DKB 901 x AG 122, XB 7012 x DKB 333, XB 7012 x P 3041, DKB 333 x P 3041, P 3041 x BR 106, P 3041 x BR 106 e BR 106 xAG 122.

Em baixo P e N, a CEC foi significativa para P100 e IC. Por causa da maior correlação genética positiva entre IC e RG, essa característica apresentou-se como a mais promissora para auxiliar na seleção, visando ao aumento da média de RG. Almeida et al. (2003) compararam híbridos de milho e observaram que houve variação genética para RG e para IC e verificaram que pode ser possível aumento no RG, por meio do incremento no IC. As combinações híbridas AG 1051 x P 3041 e AG 1051 x DKB 747 foram as únicas que simultaneamente tiveram estimativas positivas de CEC para IC e RG estatisticamente iguais às do genótipo de melhor rendimento neste ambiente.

\section{CONCLUSÕES}

A capacidade específica de combinação do rendimento de grãos não é importante quando existe estresse de nitrogênio ou fósforo.

A capacidade geral de combinação do rendimento de grãos somente não é importante em alto fósforo e baixo nitrogênio.

As correlações genéticas entre características secundárias são mais diferenciadas pelos estresses de fósforo do que pelo nitrogênio.

\section{AGRADECIMENTOS}

Ao Conselho Nacional de Desenvolvimento Científico e Tecnológico, Coordenação de Pessoal de Nível Superior e à Fundação de Amparo à Pesquisa do Estado de Minas Gerais, pelo suporte financeiro, e aos alunos e técnicos do PROGRAMA MILHO® UFV, na condução dos experimentos.

\section{REFERÊNCIAS}

Almeida ML, Sangoi L, Nava, IC, Galio J, Trentin OS \& Rampazzo C (2003) Crescimento inicial de milho e sua relação com o rendimento de grãos. Ciência Rural, 33:189-194.

Alves VMC, Novaes RF, Oliveira MFG \& Barros NF (1996) Efeito da omissão de fósforo na absorção de nitrogênio por híbridos de milho (Zea mays, L.). Revista Ceres, 43: 435-443.

Alves VMC, Vasconcellos CA, Freire FM, Pitta GVE, França GE, Rodrigues Filho A, Araújo JM, Vieira JR \& Loureiro JE (1999) Milho. In: Ribeiro AC, Guimarães PTG \& Alvarez VVH (Eds.) Recomendações para o uso de corretivos e fertilizantes em Minas Gerais - $5^{\text {a }}$ Aproximação. Viçosa, Comissão de Fertilidade do Solo do Estado de Minas Gerais. p.314-316.
Banziger M \& Betran FJ (1997) Efficiency of high nitrogen selection environments for improving maize for low nitrogen target environments. Crop Science, 37:1103-1109.

Banziger M \& Lafitte HR (1997) Efficiency of secondary traits for improving maize for low-nitrogen target environments. Crop Science, 37:1110-1117.

Betran FJ, Beck D, Banziger M \& Edmeades GO (2003a) Genetic analysis of inbred and hybrid grain yield under stress and nonstress environments in tropical maize. Crop Science, 43:807-817.

Betran FJ, Beck D, Banziger M \& Edmeades GO (2003b) Secondary traits in parental inbreds and hybrids under stress and non-stress environments in tropical maize. Field Crops Research, 83:51-65

Coimbra RR, Martins ECA, Miranda GV, Naoe LK, Cardoso EA \& Archangelo ER (2008) Capacidade de combinação de genótipos de milho para solos com baixos níveis de fertilidade. Revista de Ciências Agrárias, 50:23-33.

Cruz CD (2006) Programa genes: Biometria. Viçosa, Universidade Federal de Viçosa. 383p.

Fidelis RR, Miranda GV, Santos IC, Galvão JCC, Peluzio JM \& Lima SO (2007) Fontes de germoplasma de milho para estresse de baixo nitrogênio. Pesquisa Agropecuária Tropical, 37:147153.

Galvão JCC \& Miranda GV (2004) Tecnologias de Produção de Milho. $1^{\text {a }}$ Ed. Viçosa, Editora UFV. 336p.

Gama EEG, Teixeira FF, Parentoni SN, Guimarães PEO, Pacheco CAP, Alves VM, Meirelles WF \& Leite CEP (2006) Genetic variability in inbred lines from a tropical maize germoplasm under selection for phosphorus efficiency uptake. In: XXVI Congresso Nacional de Milho e Sorgo, Belo Horizonte. Anais, CD-ROM.

Griffing B (1956) Concept of general and specific combining ability in relation to diallel crossing systems. Australian Journal of Biological Sciences, 9:463-493.

Machado JC, Souza JC, Ramalho MA \& Lima JL (2008) Estabilidade de produção de híbridos simples e duplos de milho oriundos de um mesmo conjunto gênico. Bragantia, 67:627-631.

Médici LO, Pereira MB, Lea PJ \& Azevedo RA (2004) Diallel analysis of maize lines with contrasting responses to applied nitrogen. Journal of Agricultural Science, 142:535-541.

Miranda GV, Godoy Cl, Souza LV \& Santos IC (2005a) Selection of discrepant maize genotypes for nitrogen use efficiency by a chlorophyll meter. Crop Breeding and Applied Biotechnology, 5:451-459.

Miranda GV, Souza LV, Coimbra RR, Galvão JCC, Vaz De Melo A, Guimarães LJM \& Vilela FO (2005b) Comportamento de cultivares de milho em Minas Gerais Safras 1998-1999 e 19992000. Revista Ceres, 301:401-419.

Miranda GV, Souza IV, Galvão JCC, Guimarães LJM, Vaz de Melo A \& Santos IC (2008) Genetic variability and heterotic groups of brazilian popcorn populations. Euphytica, 162:431-440.

Miranda GV, Souza LV, Guimarães LJM , Namorato H, Oliveira LR \& Soares MO (2009) Multivariate analyses of genotype $\mathrm{x}$ environment interaction of popcorn. Pesquisa Agropecuária Brasileira, 44:45-50.

Novais RF \& Smyth TJ (1999) Fósforo em solo e planta em condições tropicais. Viçosa, Universidade Federal de Viçosa. 399 p.

Novais RF, Ferreira RP, Neves JCL \& Barros NF (1985) Absorção de fósforo e crescimento do milho com sistema radicular parcialmente exposto à fonte de fósforo. Pesquisa Agropecuária Brasileira, 20:749-754. 
Presterl T, Groh S, Landbeck M, Seitz G, Schmidt W \& Geiger HH (2002) Nitrogen uptake and utilization efficiency of European maize hybrids developed under conditions of low and high nitrogen input. Plant Breeding, 121:480-486.

Presterl T, Seitz G, Landbeck M, Thiemt EM, Schmidt W \& Geiger HH (2003) Improving nitrogen use efficiency in Europen maize: estimation of quantitative genetic parameters. Crop Science, 43:1259-1265

Ribaut JM, Fracheboud Y, Monneveux P, Banziger M, Vargas M \& Jiang C (2007) Quantitative trait loci for yield and correlated traits under high and low soil nitrogen conditions in tropical maize. Molecular Breeding, 20:15-29.

Rodrigues F, Von Pinho RG, Albuquerque CJB, Faria Filho EM \& Goulart EM (2009) Capacidade de combinação entre linhagens de milho visando à produção de milho verde. Bragantia, 68:7584.
Silva RG, Miranda GV, Cruz CD, Galvão JCC \& Silva DG (2008) Potencial genético das populações de milho UFVM 100 e UFVM 200 avaliadas em solos com deficiência de nitrogênio. Revista Caatinga, 21:22-29.

Scapim CA, Carvalho CGP \& Cruz CD (1995) Uma proposta de classificação dos coeficientes de variação para a cultura do milho. Pesquisa Agropecuária Brasileira, 30:683-86.

Souza ARR, Miranda GV, Pereira MG \& Souza LV (2009) Predicting the genetic gain in the Brazilian white maize landrace. Ciência Rural, 39:19-24.

Sprague GF \& Tatum LA (1942) General and specific combining ability in single crosses of corn. Journal of American Society of Agronomy, 34:923-932.

Zhu J, Kaeppler SM \& Lynch JP (2005) Mapping of QTL controlling root hair lengh in maize (Zea mays L.) under phophurus deficiency. Plant and Soil, 270:299-310. 